Research Article

\title{
Evaluation of hypolipidemic effect of Tinospora cordifolia in cholesterol diet induced hyperlipidemia in rats
}

\author{
Sparshadeep E. M. ${ }^{1}$, Roopa P. Nayak ${ }^{2}$, Kavana G. V. ${ }^{1}$, Mohandas Rai ${ }^{3}$
}

\begin{abstract}
${ }^{1}$ Department of Pharmacology, Academy of Medical Sciences, Kannur, Kerala, India

${ }^{2}$ Department of Pharmacology, Yenepoya Medical College, Mangalore, Karnataka, India ${ }^{3}$ Department of Pharmacology, A. J. Institute of Medical Sciences, Mangalore, Karnataka, India
\end{abstract}

Received: 13 June 2016 Accepted: 29 June 2016

\begin{abstract}
* Correspondence to: Dr. Sparshadeep E. M., Email: dr.sparsha.deep@ gmail.com
\end{abstract}

Copyright: (C) the author(s), publisher and licensee Medip Academy. This is an openaccess article distributed under the terms of the Creative Commons Attribution NonCommercial License, which permits unrestricted noncommercial use, distribution, and reproduction in any medium, provided the original work is properly cited.

\begin{abstract}
Background: There is always a need for developing novel drugs with higher efficacy and fewer side effects. Though statins are generally well-tolerated drugs for hyperlipidemia with high efficacy they are not free from adverse effects. Herbal drugs are well known for their cost-effectiveness and minimal side effects. Tinospora cordifolia ( $T$. cordifolia) is one such plant with known hypolipidemic activity and wide availability in India. Hence this study is an attempt to verify and evaluate the extent of efficacy of $T$. cordifolia as a hypolipidemic agent. The objective of the study is to compare the hypolipidemic activity of aqueous root extract of $T$. cordifolia with that of Rosuvastatin in cholesterol diet-induced hyperlipidemia in rats.

Methods: Hyperlipidemia was induced in male albino rats of wistar strain in the first 30 days of feeding period and continued in the next 30 days of treatment period. Aqueous root extract of $T$. cordifolia $(2.5$ and $5 \mathrm{~g} / \mathrm{kg}$, per oral) was administered as test drug in the treatment period. Rosuvastatin $(10 \mathrm{mg} / \mathrm{kg}$, per oral) was used as the standard drug. Serum lipid profile, atherogenic index and body weights were estimated for all rats on the day before the start of the feeding period and on day 0,15 and 30 of the treatment period. The results were analyzed statistically using student's unpaired and paired t-test wherever applicable.

Results: Serum lipid levels showed significant reduction $(\mathrm{p}<0.001)$ in TC,TG, LDL-C and VLDL-C with significant elevation ( $\mathrm{p}<0.001$ ) of HDL-C in both the rosuvastatin and test groups, but the percentage reduction in lipid levels, percentage elevation of HDL-C and percentage protection from atherosclerosis was higher in rosuvastatin group than in test groups.

Conclusions: T.cordifolia has a definite hypolipidemic potential. Although its effectiveness is lesser than rosuvastatin its beneficial role as hypolipidemic agent may be tested in clinical studies.
\end{abstract}

Keywords: Cholesterol, Hyperlipidemia, Hypolipidemic, Rosuvastatin, Tinospora cordifolia

\section{INTRODUCTION}

Hyperlipidemia is a major cause of atherosclerosis and atherosclerosis-associated conditions, such as coronary heart disease (CHD), ischemic cerebrovascular disease, and peripheral vascular disease. ${ }^{1}$ An overwhelming number of studies in the past decades have established dyslipidemia as the major risk factor for the development of atherosclerotic cardiovascular disease (CVD). ${ }^{2}$ The reported prevalence of CHD in India in adult surveys has risen 4-fold over the last 40 years (to a present level of around $10 \%$ ) and even in rural areas the prevalence has doubled over the past 30 years (to a present level of around $4 \%){ }^{3}$

The development of pharmacological interventions in particular, statins has been a major contributor to reduction of disease. Statins are now used by millions of people worldwide and their dramatic effect on cardiovascular disease has been shown in a large number 
of trials and also in meta-analyses. ${ }^{2}$ Although statins are generally extremely well tolerated, statin intolerance does occur in some patients and requires careful consideration. In addition, patients are sometimes concerned about the potential risk of statins causing diabetes mellitus, cancer, and memory loss. ${ }^{4}$ Its safety has attached a great concern from scientists and researchers, such as transaminase and creatinine elevation, skeletal muscle pain and creatine kinase elevation. Therefore, developing novel classes of hypolipidemic agents which possess high efficiency and fewer adverse effects has still been a focus on the treatment of dyslipidemia. ${ }^{5}$

Medicinal plants continue to play a central role in the healthcare system of large proportions of the world's population. The advantages of herbal medicines reported are effectiveness, safety, affordability and acceptability. ${ }^{6}$ Tinospora Cordifolia (T.Cordifolia) is one such Indian medicinal plant which has been used in ayurvedic preparations through the centuries and is distributed throughout the tropical region of India. It has been shown to have hypolipidemic property in many experiments in diabetes-induced rats and rabbits. But very few reports are seen in non-diabetic rats. T. Cordifolia is already proven to be hepato-protective. Moreover this plant is cost-effective, easily available and less toxic. ${ }^{7}$ Therefore, this plant has been chosen to attempt to verify and evaluate the extent of its efficacy as a lipid lowering agent in cholesterol diet-induced hyperlipidemia in rats.

\section{METHODS}

The current study was conducted in the Central Animal House, department of pharmacology, A. J. Institute of Medical Sciences (AJIMS). The study was undertaken to evaluate hypolipidemic activity of aqueous root extract of T. cordifolia in cholesterol diet-induced hyperlipidemia in rats.

\section{Preparation of the extract}

The fresh roots of T.cordifolia were collected from Dakshina, Kannada district, Karnataka, India. Identification and extraction of the plant was done at the Srinivas College of Pharmacy, Mangalore, India. The roots of $T$. cordifolia were isolated, chopped into small pieces and dried under shade at room temperature for seven days. The dried roots were powdered and this powder was used for the preparation of aqueous extract by heat distillation process as detailed by Agarwal et al. ${ }^{8}$ The extract was assigned a code name TCrE.

\section{Animals}

Animals were procured from central animal house of department of pharmacology, AJ Institute of Medical Sciences, Mangalore, India. Healthy male albino rats of wistar strain (18-weeks-old), weighing 180-200 gm were used in the present study. They were inbred and grown under suitable laboratory conditions. They were housed two per cage in a room maintained at $12 \mathrm{~h}$ light-dark cycles and a constant temperature of $22 \pm 20{ }^{\circ} \mathrm{C}$. The animals were provided with pellet chow and water ad libitum, except during experimentation. Animal experiments were conducted upon approval from the institutional animal ethics committee, AJIMS according to the CPCSEA guidelines of animal care.

\section{Grouping}

The method described by Ghule et al was employed in the study. ${ }^{9}$ Thirty healthy male Albino rats with similar body weight were selected. They were randomised into treatment and control groups. All rats were allowed a one-week acclimatization period to become accustomed to the laboratory conditions. Rats were randomly divided into five groups, each comprising six rats.

\section{Duration of the study}

The duration of the study was for 2 months which included 30 days of feeding period and next 30 days of treatment period with continued feeding. ${ }^{9}$

\section{Feeding period}

Group I served as normal control and was fed with standard rat chow throughout the study. Group II, III, IV and $\mathrm{V}$ were fed with high-fat diet for 30 days during the feeding period and then continued the same for the next 30 days of treatment period. Rats were supplied food and water ad libitum. ${ }^{9}$

\section{Treatment period}

- Group I served as normal control (N) and received normal saline $(5 \mathrm{ml} / \mathrm{kg}$, per oral) daily for 30 days.

- Group II served as hyperlipidemic control (H) and received normal saline $(5 \mathrm{ml} / \mathrm{kg}$, per oral) daily for 30 days.

- Group III served as standard drug control (R) and received Rosuvastatin (10 mg/kg, per oral) daily for 30 days.

- Group IV served as test group $\left(\mathrm{T}_{\mathrm{A}}\right)$ and received $\mathrm{TCrE}(2.5 \mathrm{~g} / \mathrm{kg}$, per oral) daily for 30 days.

- Group V served as test group $\left(\mathrm{T}_{\mathrm{B}}\right)$ and received $\mathrm{TCrE}(5.0 \mathrm{~g} / \mathrm{kg}$, per oral) daily for 30 days.

\section{Induction of hyperlipidemia}

High cholesterol diet (HCD) comprised the following ingredients: cholesterol 5 g (Himedia Pvt Ltd, Mumbai), deoxycholic acid 5 g (Sigma-Aldrich Pvt Ltd, Mumbai), coconut oil $300 \mathrm{ml}$ (300 g), and standard rat chow $700 \mathrm{~g}$. Deoxycholic acid (5 g) was mixed thoroughly with $700 \mathrm{~g}$ of powdered rat chow diet. Simultaneously cholesterol $(5 \mathrm{~g})$ was dissolved in $300 \mathrm{ml}$ of warm coconut oil. This oil solution of cholesterol was added slowly into the powdered mixture and thoroughly mixed to obtain soft 
homogenous cakes. These cakes were daily supplied to rats in each cage in sufficient quantities. ${ }^{10}$

\section{Body weight}

Body weights of all rats were checked on the day before the start of feeding period and on day 0,15 and 30 of the treatment period. ${ }^{9}$ Total weight gain was calculated as:

Total weight gain on day $30=$ Final body weight - Initial body weight

\section{Drug treatment}

Daily single dosage of TCrE (dissolved in normal saline, $5 \mathrm{ml} / \mathrm{kg}$ ) was given orally for 30 days in the treatment period to the test groups through oral gavage procedure. The control groups received normal saline alone. The standard group received rosuvastatin $10 \mathrm{mg} / \mathrm{kg} / \mathrm{day}$ orally dissolved in $5 \mathrm{ml} / \mathrm{kg}$ normal saline. Doses of TCrE and rosuvastatin (Reddy labs Pvt Ltd, Hyderabad) were selected based on the reports in previous study which had hypolipidemic activity.,10,11 All the doses were administered between 10-11 am.

\section{Blood sampling}

All blood samples were collected within a one-hour period between 8:00 am and 9:00 am. Twelve hours fasted blood samples were collected under light ether anaesthesia by retro orbital puncture. Blood samples were collected on the day before the start of feeding period and on day 0,15 and 30 of the treatment period. These blood samples were used for serum lipid analysis. ${ }^{9}$

\section{Serum lipid analysis}

Serum lipid profile were analyzed for all rats on the day before the start of feeding period and on day 0,15 and 30 of the treatment period. Blood samples were allowed to clot for 30 minutes and serum was separated by centrifugation at 3,000 revolutions per minute (rpm) for 5 minutes in Remi centrifuge (INCO, Chennai) and transferred to sterile $1.5 \mathrm{~mL}$ centrifuge tubes. ${ }^{9}$

Serum total cholesterol (TC), triglycerides (TG) and high density lipoprotein (HDL-C), low density lipoproteins (LDL-C), very low density lipoproteins (VLDL-C) were determined by endpoint colorimetric analysis using commercial kits and autoanalyser (Lablife Robochem, RFCL Ltd.) according to the manufacturer directions.

Diagnostic reagent kit (RFCL Ltd, Dehradun) was used for estimation of triglycerides, total cholesterol and HDL$\mathrm{C}$ which used enzymatic glycerol-3-phosphate oxidase (GPO-ESPAS) and Cholesterol oxidase/peroxidase (CHOD-PAP) method. ${ }^{12}$ VLDL-C was calculated as onefifth the level of TG using empirical equation of Friedwald. ${ }^{13}$ The Friedewald method was used to calculate the LDL-C levels, which subtracts HDL-C and very low-density lipoprotein cholesterol (VLDL-C) from TC. ${ }^{13}$

$$
\mathrm{VLDL}=\mathrm{TG} / 5
$$

$\mathrm{LDL}=\mathrm{TC}-(\mathrm{HDL}+\mathrm{VLDL})$

Percentage change from initial values (day 0 of treatment period) of serum lipid levels and body weights were calculated on day 15 and day 30 of treatment period using formula: Percentage change $=[($ difference in lipid levels/day0 lipid levels) X 100]

Atherogenic index (AI) was calculated as $\mathrm{AI}=($ total serum cholesterol/total serum HDL). ${ }^{14}$

Percentage protection from atherosclerosis was calculated as Protection $(\%)=[($ difference in AI between control and treated group/AI of control) X 100]

\section{Statistical analysis}

Results were expressed as mean+standard deviation (SD) of six values $(n=6)$ for each group. Statistical differences between the controls and the treatment groups were evaluated by using student's unpaired and paired t-test wherever applicable using SPSS software package. Values were considered significant at $\mathrm{p}<0.05$ and $\mathrm{p}<0.01$; highly significant at $\mathrm{p}<0.001$.

\section{RESULTS}

Following were the effects seen in rats following administration of aqueous root extract of T.cordifolia $(2.5 / 5 \mathrm{~g} / \mathrm{kg}$, p.o., once daily) and rosuvastatin $(10 \mathrm{mg} / \mathrm{kg}$, per oral, once daily).

\section{Effect on serum lipid levels}

\section{Comparison with normal group}

On day 0 of treatment period the hyperlipidemic control group, rosuvastatin and test groups showed significant increase ( $\mathrm{p}<0.001)$ in total cholesterol, triglyceride, LDL$\mathrm{C}$, VLDL-C levels and significant decrease in HDL-C $(\mathrm{p}<0.05)$ levels (Table1, Figure1).

\section{Comparison with hyperlipidemic group}

On day 30 of treatment period Rosuvastatin group, Test group A and Test group B showed a significant decrease $(\mathrm{p}<0.001)$ in total cholesterol, triglyceride, LDL-C, VLDL-C levels and a significant increase $(\mathrm{p}<0.001)$ in HDL-C levels. (Table 1, Figure 2).

Comparison with day 0 of treatment period (intragroup)

A highly significant decrease in total cholesterol, triglyceride, LDL, VLDL levels and a significant increase 
in HDL levels was observed in rosuvastatin group and both test groups on day15 $(\mathrm{p}<0.001)$ and day 30 (p <0.001) in the same group (Table 1, Figure 1, Figure 2).

\section{Percentage change in lipid levels}

On day 30 when test groups were compared with rosuvastatin group they showed lesser percentage reduction in the serum total cholesterol, triglyceride, LDL-C and VLDL-C but test group B showed greater percentage elevation in the serum HDL-C than rosuvastatin group (Table 2 ).

\section{Effect on atherogenic index (AI)}

On day 30 the hyperlipidemic group showed a significant increase $(\mathrm{p}<0.001)$. The rosuvastatin and the test groups both reduced AI significantly $(\mathrm{p}<0.001)$. But the percentage protection against atherogenesis was greatest in the rosuvastatin group than in the test groups (Table 3).

Table 1: Serum lipid levels and atherogenic index (AI) in various groups on Day 0,15 and 30.

\begin{tabular}{|c|c|c|c|c|c|c|c|}
\hline Groups & Day & C (mg/dl) & TG (mg/dl) & HDL-C (mg/dl) & VLDL-C (mg/dl) & LDL-C (mg/dl) & $\mathbf{A I}$ \\
\hline \multirow{3}{*}{$\mathbf{N}$} & $0^{\mathrm{al}}$ & $104.93 \pm 14.18$ & $83.32 \pm 8.13$ & $40.83 \pm 2.91$ & $16.66 \pm 1.63$ & $47.43 \pm 16.12$ & $2.60 \pm 0.53$ \\
\hline & $15^{\mathrm{a} 2}$ & $104.92 \pm 13.59$ & $79.50 \pm 8.71$ & $41.26 \pm 2.18$ & $15.89 \pm 1.74$ & $47.77 \pm 11.65$ & $2.54 \pm 0.30$ \\
\hline & $30^{\mathrm{a3}}$ & $100.77 \pm 8.94$ & $79.49 \pm 7.99$ & $40.93+4.81$ & $15.35 \pm 1.21$ & $44.49 \pm 10.55$ & $2.50 \pm 0.42$ \\
\hline \multirow{3}{*}{$\mathbf{H}^{*}$} & $0^{\mathrm{bl}}$ & $293.80 \pm 18.89$ & $213.18 \pm 15.96$ & $40.29 \pm 2.91$ & $42.62 \pm 3.18$ & $206.47 \pm 19.18$ & $6.69 \pm 0.95$ \\
\hline & $15^{\mathrm{b} 2}$ & $292.04 \pm 18.95$ & $211.97 \pm 11.35$ & $36.77 \pm 4.38$ & $42.39 \pm 2.27$ & $206.69 \pm 21.02$ & $6.89 \pm 0.99$ \\
\hline & $30^{\mathrm{b3}}$ & $282.23 \pm 15.15$ & $207.81 \pm 9.52$ & $38.01 \pm 4.43$ & $41.56 \pm 1.90$ & $199.16 \pm 18.36$ & $6.83+0.72$ \\
\hline \multirow{3}{*}{$\mathbf{R}^{\#}$} & $0^{\mathrm{cl}}$ & $292.28+13.88$ & $215.57 \pm 10.32$ & $42.29+4.97$ & $43.11+2.06$ & $206.88+12.88$ & $6.99+0.83$ \\
\hline & $15^{\mathrm{c} 2}$ & $196.53 \pm 15.67$ & $158.27 \pm 16.02$ & $50.78 \pm 2.33$ & $31.65 \pm 3.20$ & $99.62 \pm 15.00$ & $3.03 \pm 0.39$ \\
\hline & $30^{\mathrm{c} 3}$ & $169.33 \pm 15.74$ & $117.05 \pm 11.08$ & $55.97 \pm 4.10$ & $23.41 \pm 2.22$ & $70.70 \pm 15.13$ & $2.26 \pm 0.25$ \\
\hline \multirow{3}{*}{$\mathbf{T}_{\mathbf{A}}{ }^{\#}$} & $0^{\mathrm{dl}}$ & $295.67 \pm 16.41$ & $204.15 \pm 12.52$ & $43.14 \pm 7.17$ & $40.83+2.50$ & $211.70+19.65$ & $7.02 \pm 1.24$ \\
\hline & $15^{\mathrm{d} 2}$ & $272.29 \pm 13.28$ & $186.82 \pm 13.87$ & $48.49 \pm 4.82$ & $37.36 \pm 2.77$ & $186.44 \pm 14.66$ & $5.65 \pm 0.53$ \\
\hline & $30^{\mathrm{d} 3}$ & $256.50 \pm 12.93$ & $169.70 \pm 15.34$ & $53.30 \pm 2.60$ & $33.94 \pm 3.07$ & $169.26 \pm 14.40$ & $4.82 \pm 0.30$ \\
\hline \multirow{3}{*}{$\mathbf{T}_{\mathbf{B}}{ }^{\#}$} & $0^{\mathrm{el}}$ & $297.80+14.26$ & $202.42+13.28$ & $39.12+4.31$ & $40.48+2.66$ & $218.19+14.19$ & $7.69+0.96$ \\
\hline & $15^{\mathrm{e} 2}$ & $245.08 \pm 19.90$ & $170.03 \pm 16.16$ & $52.49 \pm 2.47$ & $34.01 \pm 3.23$ & $158.59 \pm 19.02$ & $4.68 \pm 0.49$ \\
\hline & $30^{\mathrm{e} 3}$ & $222.95+19.48$ & $146.50 \pm 11.28$ & $60.57 \pm 3.08$ & $29.30+2.26$ & $131.31 \pm 19.54$ & $3.60 \pm 0.44$ \\
\hline
\end{tabular}

Data expressed as Mean \pm SD of 6 observations.

Student's unpaired t-test:

* Values are statistically highly significant at $\mathrm{p}<0.001$ when compared to normal control $(\mathrm{N})$

" Satistically highly significant at $\mathrm{p}<0.001$ when compared to hyperlipidemic control $(\mathrm{H})$

\section{Student's paired t-test:}

(a1 and a2), (a1 and a3): No significant difference, p>0.05; (b1 and b2), (b1 and b3): No significant difference, p >0.05; (c1 and c2), (c1 and $\mathrm{c} 3)$ :Values are statistically significant at $\mathrm{p}<0.001$; (d1 and d2), (d1 and d3):Values are statistically significant at $\mathrm{p}<0.001$; (e1 and $\mathrm{e} 2),(\mathrm{e} 1$ and e3):Values are statistically significant at $\mathrm{p}<0.001$

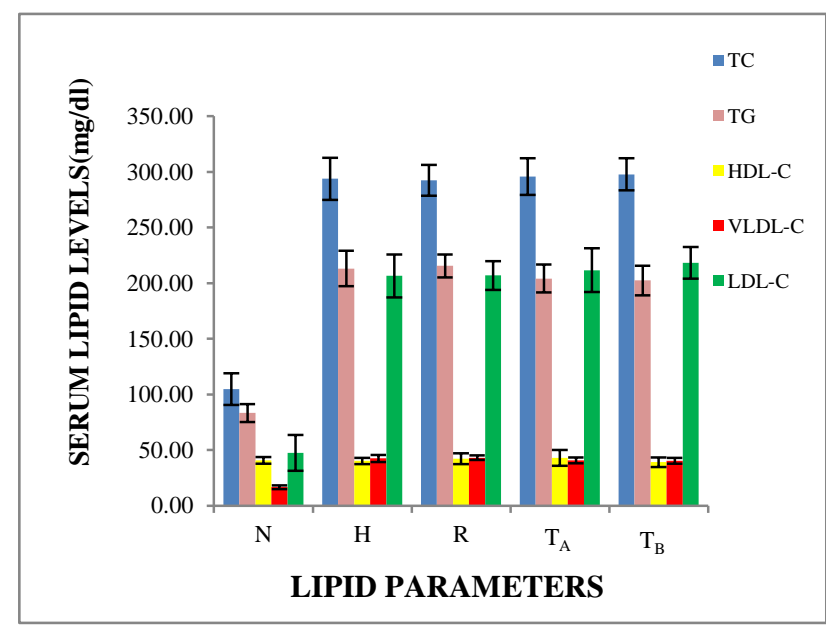

Figure 1: Serum lipid levels on day 0.

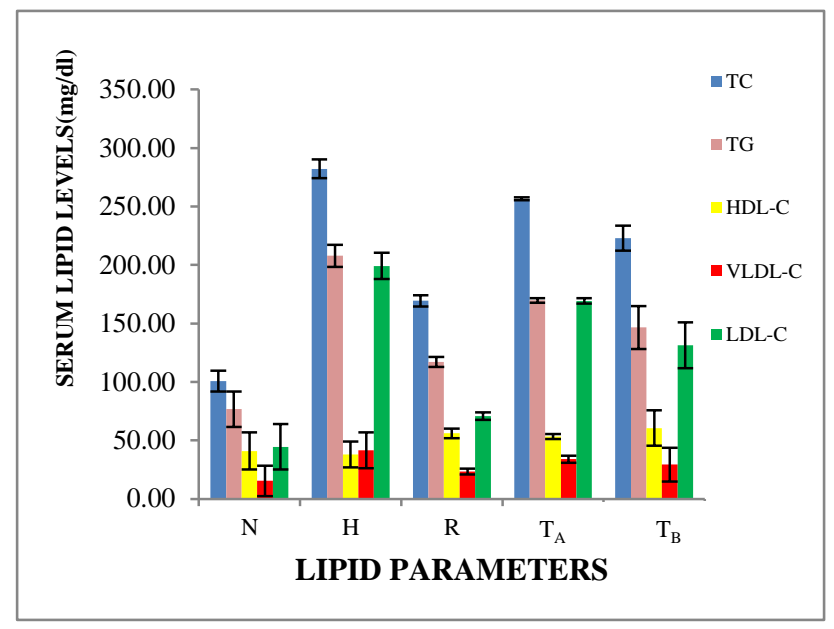

Figure 2: Serum lipid levels on day 30. 
Table 2: Mean percentage change (\%) from day 0 values of serum lipid levels in different groups on day 15 and 30.

\begin{tabular}{|c|c|c|c|c|c|c|}
\hline \multirow{2}{*}{ Groups } & \multirow{2}{*}{ Day } & \multicolumn{5}{|c|}{ Mean percentage change $(\%)$} \\
\hline & & C & TG & HDL-C & VLDL-C & LDL-C \\
\hline \multirow{2}{*}{$\mathbf{N}$} & 15 & 0.21 & 4.54 & 1.45 & 4.60 & 4.64 \\
\hline & 30 & 3.02 & 7.69 & 0.03 & 7.69 & 1.25 \\
\hline \multirow{2}{*}{$\mathbf{H}$} & 15 & 0.55 & 6.65 & 8.12 & 0.32 & 0.12 \\
\hline & 30 & 3.83 & 2.29 & 5.35 & 2.26 & 3.49 \\
\hline \multirow{2}{*}{$\mathbf{R}$} & 15 & 32.80 & 26.66 & 21.59 & 26.66 & 51.96 \\
\hline & 30 & 42.17 & 45.74 & 33.59 & 45.74 & 66.04 \\
\hline \multirow{2}{*}{$\mathbf{T}_{\mathbf{A}}$} & 15 & 7.84 & 8.49 & 13.56 & 8.49 & 11.74 \\
\hline & 30 & 13.19 & 16.84 & 25.74 & 16.84 & 19.91 \\
\hline \multirow{2}{*}{$\mathbf{T}_{\mathbf{B}}$} & 15 & 17.78 & 16.05 & 35.21 & 16.05 & 27.39 \\
\hline & 30 & 25.22 & 27.62 & 56.11 & 27.62 & 39.94 \\
\hline
\end{tabular}

Data expressed as mean of 6 observations.

\section{Effect on weight gain}

On day 30 the hyperlipidemic group showed a significant increase $(\mathrm{p}<0.01)$ while the rosuvastatin and the test group-B showed a significant decrease $(\mathrm{p}<0.001)$ in weight gain. But the test group-A showed no significant decrease $(p>0.05)$ in weight gain (Table 4$)$.

Table 3: Atherogenic index (AI) in different groups on day 30 .

\begin{tabular}{|lll|}
\hline Groups & Atherogenic index & Protection (\%) \\
\hline $\mathbf{N}$ & 2.50 & - \\
\hline $\mathbf{H}$ & 7.5 & - \\
\hline $\mathbf{R}$ & 3.03 & 59.55 \\
\hline $\mathbf{T}_{\mathbf{A}}$ & 4.82 & 35.75 \\
\hline $\mathbf{T}_{\mathbf{B}}$ & 3.60 & 50.85 \\
\hline
\end{tabular}

Data expressed as mean of 6 observations.

Table 4: Weight gain in different groups on day 30 as compared to day 0 .

\begin{tabular}{|ll|}
\hline Groups & Weight gain $(\mathrm{gm})$ \\
\hline $\mathbf{N}$ & $6.66 \pm 2.58$ \\
\hline $\mathbf{H}$ & $14.16 \pm 3.76^{*}$ \\
\hline $\mathbf{R}$ & $7.5 \pm 2.73^{\#}$ \\
\hline $\mathbf{T}_{\mathbf{A}}$ & $10.0 \pm 4.47^{@}$ \\
\hline $\mathbf{T}_{\mathbf{B}}$ & $9.16 \pm 3.76^{\$}$ \\
\hline
\end{tabular}

Data expressed as Mean \pm SD of 6 observations. Student's unpaired $t$-test: $*$ Highly significant difference when compared

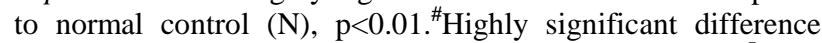
when compared to Hyperlipidemic control, $\mathrm{p}<0.01$. ${ }^{@}$ No significant difference when compared to Hyperlipidemic control, $\mathrm{p}>0.05{ }^{\$}$ Significant difference when compared to Hyperlipidemic control, $\mathrm{p}<0.05$.

\section{DISCUSSION}

It has been well established that nutrition plays an important role in the etiology of hyperlipidemias and atherosclerosis. ${ }^{1}$ This study has utilised deoxycholic acid, cholesterol, coconut oil and chow in the hyperlipidemic diet. A high-fat/high-cholesterol diet supplemented with cholic acid changes the lipoprotein profile to a more atherogenic one by increasing levels of LDL-C and lowering HDL-C. ${ }^{15}$ Many studies have shown that chow based diets supplemented with varying amounts of cholesterol and sodium cholate can induce hypercholesterolemia in mice and rats presumably by interfering with the hepatobiliary excretion of cholesterol. ${ }^{16,17}$ The mechanism of action of cholic acid is two-fold. It causes an increase in cholesterol absorption and a concomitant suppression of cholesterol $7 \alpha-$ hydroxlyase activity that results in decreased cholesterol excretion. Cholic acid improves cholesterol absorption by its emulsifying property. ${ }^{18}$

It is evident from the results that although both rosuvastatin and TCrE showed significant lipid lowering and HDL-C increasing activity, the percentage change in lipid parameter except for HDL-C was lesser with TCrE than with rosuvastatin. The explanation to this effect possibly lies in the difference in the mechanisms of hypolipidemic action of $T$. cordifolia and the rosuvastatin. Although multiple mechanisms have been suggested for T. cordifolia, none of those is suggested to affect the de novo synthesis of cholesterol like that by statins. Statins act by blocking the HMG-CoA reductase enzyme, which catalyzes the rate-limiting step in de novo cholesterol synthesis. Rosuvastatin is the most effective statin to lower LDL-C, with reductions of up to $63 \%$ reported with a daily dose of 40 miligram. ${ }^{19}$

The exact mechanism by which TCrE reduced the serum cholesterol is not clear. But the observation of increased HDL-C levels could be one of the possible mechanisms for decrease in serum lipid levels by TCrE. The increased HDL-C facilitates the transport of TG or cholesterol by a 
pathway termed 'reverse cholesterol transport' from serum to liver where it is catabolised and excreted out of the body. Increase of HDL-C is attributed to the mobilization of cholesterol from peripheral cells to the liver by the action of LCAT. ${ }^{20}$

Studies have shown the presence of flavonoids, saponins, tannins, triterpenoids, steroids and polyphenolics in root

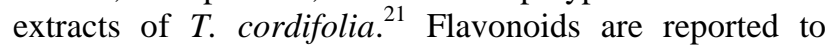
enhance the activity of lecithin cholesterol acyl transferase (LCAT) which plays a key role in the incorporation of free cholesterol into HDL causing increase in HDL-C. ${ }^{22}$ Saponins are highly active plant compounds reported to increase faecal cholesterol excretion and also increase the lipoportein lipase activity (LPL)which helps in faster removal of free fatty acid from circulation consequently decreasing total cholesterol. ${ }^{23,9}$ Tannins are reported to increase the activity of the endothelium bound lipoprotein lipase activity, which hydrolyzes triglycerides. Plant sterols are also reported to decrease cholesterol absorption and increase its excretion. $^{23}$

The TCrE also has anti-oxidant property. ${ }^{24}$ These phyto constituents would have contributed to the lipid lowering effect of TCrE in this study. But the presence of these phyto constituents in the TCrE needs to be investigated by further studies (such as phytochemical screening, fecal bile acid excretion).

According to Kannel et al, an ideal drug is one which raises HDL-C along with the lowering of LDL-C. ${ }^{25}$ Biochemical estimations showed that both the TCrE as well as rosuvastatin increased the protective HDL-C level and decreased the atherogenic LDL-C and VLDL-C levels. Although TCrE has shown greater elevation of HDL-C when compared to rosuvastatin, the percentage protection from atherosclerosis remains the similar. This is an important advantage in treatment of hypercholesterolemia particularly among Indians where low HDL-C is the most prevalent lipoprotein abnormality. ${ }^{26}$

High levels of total cholesterol and most importantly, LDL-C are the predictors of atherosclerosis. ${ }^{27}$ TCrE significantly reduced both the total cholesterol and LDLC. Recent studies show that triglycerides are directly or indirectly related to coronary heart diseases. In the present study, TCrE has markedly decreased the triglycerides level. These effects (although to a greater extent with rosuvastatin) along with significant decline in atherogenic index without causing mortality or adverse effects in rats, points towards the efficacious role of T.cordifolia as hypolipidemic agent.

The pleotropic actions and the distinct mechanism of hypolipidemic action definitely assign the statins as the clinically most efficacious hypolipidemic agents. But the significant decrease in lipid profile, elevation in the HDL, good safety margin in experimental models combined with traditional application as cardioprotective agent definitely promote T.cordifolia as an agent to be clinically tested for the treatment of hyperlipidemia and its associated cardiovascular disorders

\section{CONCLUSION}

The result shows that the $T$. cordifolia has a definite hypolipidemic and hence cardioprotective and antiatherosclerotic potential. There is also a valid scientific basis for consuming it for clinical benefits in the treatment of cardiovascular diseases in India. Hence, the present study helps to support the traditionally claimed cardioprotective activity of $T$. cordifolia. However, further studies are necessary to support these findings. Also an extensive case-control study is required to document its therapeutic application in human beings.

\section{ACKNOWLEDGEMENTS}

Authors would like to acknowledge the cooperation and support offered by the teaching and non-teaching staff of department of Pharmacology, AJIMS, Mangalore, Karnataka, India.

\section{Funding: No funding sources}

Conflict of interest: None declared

Ethical approval: The study was approved by the Institutional Ethics Committee

\section{REFERENCES}

1. Mahley RW, Bersot TP. Drug therapy for hypercholesterolemia and dyslipidemia. In: Brunton LB, Lazo JS, Parker KL, eds. Goodman and Gilman's, The Pharmacological Basis of Therapeutics. $12^{\text {th }}$ ed. New York, NY: McGraw-Hill; 2011:878-908.

2. Wiklund O, Pirazzi C, Romeo S. Monitoring of lipids, enzymes, and creatine kinase in patients on lipid-lowering drug therapy. Curr Cardiol Rep. 2013;15(9):397.

3. Reddy KS. India wakes up to the threat of cardiovascular diseases. J Am Coll Cardiol. 2007;50:1370-2.

4. Fitchett DH, Hegele RA, Verma S. Statin intolerance. Circulation 2015;131:389-91.

5. Guo M, Liu Y, Gao Z, Shi D. Chinese herbal medicine on dyslipidemia: progress and perspective. Evid Based Complement Alternat Med. 2014;163036:1-11

6. Kumar A, Sunil C, Dhaliya SA, Surya AS, Dawn VT, Carla B. A review of hyperlipidemia and medicinal plants. Int J Bio Med S. 2013;2(4):219-37.

7. Sinha K, Mishra NP, Singh J, Khanuja SPS. Tinospora cordifolia (Guduchi), a reservoir plant for therapeutic applications: A Review. Indian J Tradit Know. 2004;3(3):257-70.

8. Agarwal A, Malini S, Bairy KL, Rao MS. Effect of Tinospora cordifolia on learning and memory in 
normal and memory deficit rats. Indian J Pharmacol. 2002;34:339-49.

9. Ghule BV, Ghante MH, Saoji AN, Yeole PG. Antihyperlipidemic effect of the methanolic extract from Lagenaria Siceraria stand. Fruit in hyperlipidemic rats. J Ethnopharmacol. 2009;124:333-37.

10. Kumar V, Singh S, Kumar A, Khan M. Hypolipidemic activity of Anthocephalus indicus (kadam) in hyperlipidemic rats. Med Chem Res. 2008; $17: 152-8$.

11. Luigi S, Elisabetta G, Paolo G, Uliano G, Elena N, Benedetta C, et al. Rosuvastatin, but not simvastatin, provides end-organ protection in stroke-prone rats by antiinflammatory effects. Arterioscler Thromb Vasc Biol. 2005;25:598-603.

12. Werner M, Gabrielson DG, Eastman G. Ultra micro determinations of serum triglycerides by bioluminescent assay. Clin Chem. 1981;21:268-71.

13. Friedewald WT, Levy RI, Fredrickson DS. Estimation of the concentration of low-density lipoprotein cholesterol in plasma without use of the preparative ultracentrifuge. Clin Chem. 1972;18(6):499-562.

14. Maruthappan V, Shakthi SK. Hypolipidemic activity of haritaki (Terminalia chebula) in atherogenic diet induced hyperlipidemic rats. J Adv Pharma Tech Res. 2010;1(2):229-35.

15. Srivastava RA, Srivastava N, Averna M. Dietary cholic acid lowers plasma levels of mouse and human apolipoprotein A-I primarily via a transcriptional mechanism. Eur $\mathrm{J}$ Biochem. 2000;267(13):4272-80.

16. Jeong WI, Jeong DH, Do SH, Kim YK, Park HY, Kwon OD, et al. Mild hepatic fibrosis in cholesterol and sodium cholate diet-fed rats. J Vet Med Sci. 2005;67(3):235-42.

17. Venkatakrishnan K, Thangarajan S. Antihypercholesterolemic effect of Bacopa monniera linn. on high cholesterol diet induced hypercholesterolemia in rats. Asian Pac J Trop.2012;5(12):949-55.
18. Dhulasavant V, Shinde S, Pawar M, Naikwade NS. Antihyperlipidemic activity of Cinnamomum tamala nees on high cholesterol diet induced hyperlipidemia. Int $\mathrm{J}$ Pharm Tech Res. 2010;2(4):2517-21.

19. Rao SK, Prasad T, Mohanta GP, Manna PK. An overview of statins as hypolipidemic drugs. Int $\mathbf{J}$ Pharm Sci Drug Res. 2011;3:178-83.

20. Puri Dinesh. Lipid metabolism II: lipoproteins, cholesterol and prostaglandins. In: Bhatnagar G, eds. Text book of medical biochemistry. $3^{\text {rd }}$ edition. Haryana:Elsevier;2011:235-57.

21. Kavitha BT, Shruthi SD, Rai RS, Ramachandra YL. Phytochemical analysis and hepatoprotective properties of Tinospora cordifolia against carbon tetrachloride-induced hepatic damage in rats. J Basic Clin Pharm. 2011;2(3):139-42.

22. Cheurfa M, Allem R. Study of hypocholesterolemic activity of Algerian Pistacia lentiscus leaves extracts in vivo. Rev Bras Farmacogn. 2015;25(2):142-4.

23. Kothari S, Jain AK, Mehta SC, Tonpay SD. Hypolipidemic effect of fresh Triticumaestivum (wheat) grass juice in hypercholesterolemic rats. Acta Pol Pharm. 2011;68(2):291-4.

24. Mainzon PP, Menon VP, Gunasekaran G. Hypolipidaemic action of Tinospora cordifolia roots in alloxan diabetic rats. $\mathbf{J}$ Ethnopharmacol. 1999;64:53-7.

25. Kannel WB, Castelli WP, Gordon T. Cholesterol in the prediction of atherosclerotic disease new perspectives based on the Framingham study. Ann Intern Med. 1979;90(1):85-91.

26. Gupta R, Gupta HP, Kumar N, Joshi AK, Gupta VP. Lipoprotein lipids and prevalence of hyperlipidemia in rural India. J Cardiovasc Risk. 1994;1:179-83.

27. Temme EH, Van HPG, Schouten EG, Kesteloot H. Effects of a plant sterol-enriched spread on serum lipids and lipoproteins in mildly hypercholesterolaemic subjects. Acta Cardiol. 2002;57:111-5.

Cite this article as: Sparshadeep EM, Nayak RP, Kavana GV, Rai M. Evaluation of hypolipidemic effect of Tinospora cordifolia in cholesterol diet induced hyperlipidemia in rats. Int $\mathrm{J}$ Basic Clin Pharmacol 2016;5:1286-92. 